\title{
Radical prostatectomy for carcinoma of the prostate
}

\author{
Makoto Ohori, Michael Kattan, Peter T Scardino and Thomas M Wheeler \\ Baylor College of Medicine $\&$ Memorial Sloan Kettering Cancer Center, TX, USA
}

\begin{abstract}
Morphologic features of prostatic adenocarcinoma in the radical prostatectomy (RP) specimen are powerful prognostic indicators for prognosis for disease-free survival. This review discusses the methods of sampling of the RP specimen to optimize the detection of these morphologic features, balanced against the added expense of submitting the entire gland for sectioning. Gleason grade, one of the most powerful prognostic factors, is discussed briefly, including the percent pattern 4/5 cancer compared to the standard Gleason grading. Pathologic stage, as defined by the TNM system, is discussed in detail, both in terms of precise histological definition of each category, as well as the associated prognostic implications. Surgical margin status is also important prognostically across all pathologic stages categories. Perineural invasion, which has been used diagnostically in prostate cancer for several decades, has emerged as a very important prognostic indicator as well, as determined by the quantitative aspects of tumor in the perineural space. The effect of tumor volume on prognosis is discussed, as well as the newer concepts of the prognostic significance of zone of origin of the tumor and the presence or absence of intraductal carcinoma.

Modern Pathology (2004) 17, 349-359, advance online publication, 6 February 2004; doi:10.1038/modpathol.3800056
\end{abstract}

Keywords: prostate carcinoma; pathologic stage; radical prostatectomy

Radical prostatectomy (RP) for carcinoma of the prostate was performed and reported 100 years ago at the Johns Hopkins hospital by Dr Hugh Hampton Young who utilized a transperineal approach. He reported this and several other cases in the Bulletin of the Johns Hopkins Hospital. ${ }^{1}$ Major impediments to utilizing RP in the years that followed related primarily to the almost universal presence of impotence and less commonly incontinence following the surgery. Also a large proportion of cases were first diagnosed with stage IV disease before widespread awareness of the disease and the advent of screening with serum prostate-specific antigen (PSA) in the late 1980s. In addition, initially there were high expectations for hormonal therapy in the 1940 s and radiation therapy in the years that followed. However, it became clear very early that hormonal therapy could delay but not halt the progression of disease. Also radiotherapy was fraught with a high frequency of post-therapy positive biopsies and subsequent late recurrence; and for the latter, at times a much less differentiated tumor.

Correspondence: TM Wheeler, 6565 Fannin, MS 205, Department of Pathology, Houston, TX 77030, USA.

E-mail: twheeler@bcm.tmc.edu

Received 4 September 2003; accepted 19 November 2003; published online 6 February 2004
The resurgence of RP for prostate cancer was brought about by another surgeon at Johns Hopkins, Patrick Walsh, MD, who along with Donker, reported on the anatomical distribution of nerves to the penile corpora in the male fetus. They subsequently designed an operation (now called 'anatomical RP') that would spare these nerves, which were located in the neurovascular bundle next to the postero-lateral aspect of the prostate. ${ }^{2}$ In this paper he also reported a small series of patients he had treated by this new method of RP and reported postoperative potency rates. Although the postoperative potency rate were far from perfect, these results were much better than those seen at any time previously for patients treated by RP. Today, RP has evolved into the preferred and most commonly used treatment for relatively young men with clinically localized prostate cancer and with at least a 10 year life expectancy.

\section{Sampling methods of RP specimens}

Several papers have been published regarding the appropriate methods of histological sampling of the RP specimen. ${ }^{3-7}$ Regardless of how the prostate is sectioned, the first step is to paint the entire external surface of the prostate with an indelible ink, with different colors to denote the right and left sides. In most centers, the apical and bladder neck margins 
are removed and submitted as separate specimens. However, some centers submit one or both of these margins as shave margins en face, and would regard any tumor in this section as evidence of a positive surgical margin (+SM). Although this is a more sensitive method to detect a positive margin, it has the potential of classifying an otherwise close margin as positive. More commonly today, these margins (especially the apical) are removed as specimens of varying width, sectioned like a pie or parallel to the urethra, and submitted to examine the margins in the perpendicular plane to the ink. In this method, any tumor on ink is considered to be a $+\mathrm{SM}$. However, if there is inadvertent entry into the plane of the prostate by the surgeon, the ink may penetrate along these planes. In such cases, tumor on ink is considered to be an artifact, and the SM considered negative.

Each method of sampling of the remainder of the prostate has its own advantages and disadvantages, and should be adopted depending upon the specific needs of the institution. As a practical point, this is a very important area, in that only $12 \%$ of pathologists responding to a recent survey indicated that they processed the entire prostate for microscopic examination. ${ }^{8}$ This is in spite of the fact that one study showed a mean of only 26 blocks per specimen were required to submit the entire prostate and the lower portion of the seminal vesicles with standard blocks. ${ }^{9}$ Hollenbeck et $a 1^{10}$ compared the partial sampling to whole mount sections and concluded that there were no differences between the two methods regarding pathological outcome, including Gleason score, the frequency of extraprostatic extension, positive surgical margins or seminal vesicle invasion. However, Smith Sehdev et al $1^{11}$ studied 78 patients with clinical stage T1c PCa with adverse pathologic features to compare the findings of partial sampling (by nine different methods) to those of the entirely submitted gland (Tables 1 and 2). As they concluded in this study, pathologists can decide which sampling method to use by balancing the added expense to the risk of missing important prognostic parameters. However, it seems that all the posterior sections are necessary to be evaluated and it may not be ideal to base the method of sampling on needle biopsy findings.

At Baylor, we have used a whole-mount step sections as a routine practice. In addition to high sensitivity and specificity of the entirely embedded and sectioned gland for the detection of adverse pathologic features, additional advantages of wholemount step section technique are (1) ease of orientation of the specimen and the location of tumor foci, (2) ease of determination of the location and extent of extraprostatic extension and/or positive surgical margins, (3) ease of determination of the distribution of the various Gleason patterns for

Table 1 Partial sampling methods from Smith Sehdev et al. ${ }^{11}$ Hum Pathol 32: 494, 2001

Partial sampling methods

\begin{tabular}{ll}
\hline 1. & Every posterior section \\
2. & Every posterior section plus anterior sampling A $\mathrm{A}^{\mathrm{a}}$ \\
3. & Every posterior section plus anterior sampling B \\
4. & Every other posterior section \\
5. & Every other posterior section plus anterior sampling A \\
6. & Every other posterior section plus anterior sampling B \\
7. & Every posterior section; limit to side (R or L) with previous (+) needle biopsy \\
8. & Every posterior section plus anterior sampling A; limit to side (R or L) with previous (+) needle biopsy \\
9. & Every posterior section plus anterior sampling B; limit to side (R or L) with previous (+) needle biopsy
\end{tabular}


submitted.

${ }^{\mathrm{b}}$ Anterior sampling method B: if any posterior slide shows sizable, predominantly anterior tumor, all ipsilateral sections are submitted.

Table 2 Comparative analysis of sampling methods from Smith Sehdev et al. ${ }^{11}$ Hum Pathol 32: 494, 2001

\begin{tabular}{lcccc}
\hline Method & $\begin{array}{c}\text { Gleason score } \geq 7 \\
(\mathrm{n}=52)(\%)\end{array}$ & $\begin{array}{c}\text { Surgical margin } \\
(\mathrm{n}=14)(\%)\end{array}$ & $\begin{array}{c}\text { Extraprostatic } \\
\text { extension }(\mathrm{n}=54)(\%)\end{array}$ & $\begin{array}{c}\text { Mean slides submitted } \\
(\text { range })\end{array}$ \\
\hline 1 & 92 & 86 & 84 & $23(14-37)$ \\
2 & 98 & 100 & 96 & $27(16-49)$ \\
3 & 94 & 86 & 91 & $26(15-49)$ \\
4 & 83 & 71 & 62 & $15(10-21)$ \\
5 & 94 & 71 & 76 & $19(12-37)$ \\
6 & 89 & 79 & 71 & $17(10-37)$ \\
8 & 83 & 93 & 85 & $14(9-24)$ \\
9 & 92 & 86 & 78 & $17(10-36)$ \\
10 In entirety & 85 & 100 & 100 & $16(9-36)$ \\
\hline
\end{tabular}


each cancer focus, and, (4) ease of determination of tumor volume. ${ }^{12,13}$ These advantages are most important in an academic setting in which one wishes to have the most accurate pathologic stage, as there is no proven benefit at this time to the initiation of adjuvant therapy prior to biochemical recurrence in those patients with adverse pathologic features in the RRP specimen. On the other hand, the principal disadvantages of the whole mount technique are the increased technical cost (increased technician time and requirement of a separate, longer cycle tissue processor run) and the fact that the slides and blocks cannot be stored in the traditional manner. In addition, some have suggested that whole-mount sections are not as sensitive as traditional sections to detect EPE and $+\mathrm{SM}$, because the thickness of the slices of the prostate to prepare whole-mount sections are generally thicker than those of standard sections. However, no special microtome is required; most existing microtomes can easily accommodate the larger whole-mount blocks, although an occasional very large prostate must be divided into right and left sides prior to sectioning.

An example of fresh tissue sampling for research is shown in Figure 1. At Baylor, following informed consent of the patient, we routinely harvest fresh prostate tissue in all cases of RP for prostate cancer, radical cystoprostatectomy for bladder cancer and suprapubic prostatectomy for nodular hyperplasia, and the entire prostate in organ donor cases. ${ }^{14} \mathrm{~A}$ pathologist or trained technician harvests the tissue in the operating room with the least possible delay. In most cases, each individual sample is immediately snap frozen in liquid nitrogen and later transferred to a $-80^{\circ} \mathrm{C}$ freezer. The fresh prostate from radical prostatectomy or cystoprostatectomy is placed on a cutting table and a single cut is made through the mid portion beginning on the posterior surface of the prostate in a plane perpendicular to the rectal surface. The surgical margins are not

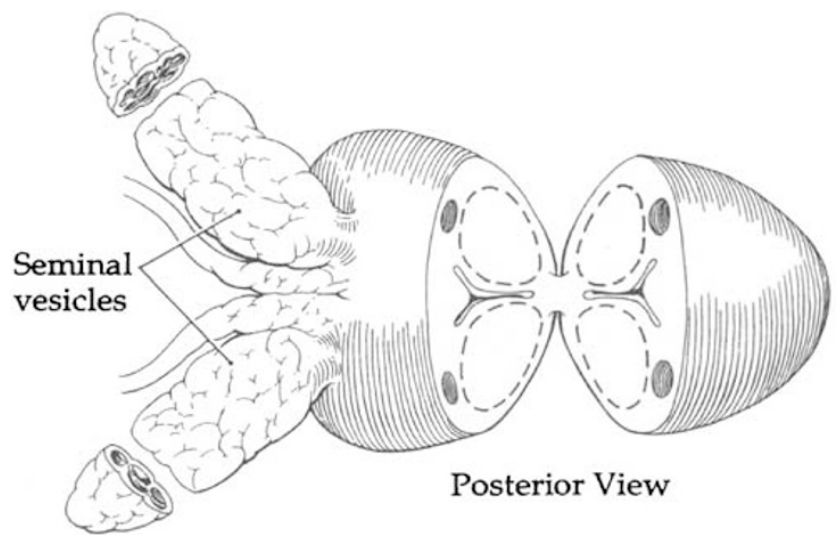

Figure 1 View of the prostate and attached seminal vesicles after a single slide has been made through the mid-prostate posteriorly. The harvest includes four quadrants of peripheral zone (punches), four quadrants of transition zone (dashed lines, may be punched or shaved), and tips of the seminal vesicles. inked prior to the sectioning. Both halves are left attached by the anterior fibromuscular stroma. This procedure exposes two 'mirror image' halves of the prostate from which at least 10 separate specimens are harvested. Additional samples of tumor may be obtained if the tumor is large and grossly visible. The peripheral zone samples are taken with a $6 \mathrm{~mm}$ punch biopsy instrument commonly used by dermatologists to obtain skin biopsies. These instruments are available as sterile disposables in sizes of 2,3,4,5, and $6 \mathrm{~mm}$ diameter (Acu-Punch ${ }^{\mathbb{}}$, Acuderm, Inc., Fort Launderdale, FL, USA). Approximately 150$200 \mathrm{mg}$ of tissue can be obtained with the $6 \mathrm{~mm}$ punch, which is our preferred biopsy instrument. For the peripheral zone, care is taken to take the punch at least $2 \mathrm{~mm}$ inside the capsule so as not to interfere with subsequent pathologic staging. For the transition zone, two methods of sampling are used. Many of the prostatectomies contain significant amounts of nodular hyperplasia. In these instances, the tissue bulging from the cut surface is shaved off, permitting the procurement of $0.5-2 \mathrm{~g}$ per each of four separate samples. When no nodular hyperplasia is present, the samples are obtained with the punch biopsy device. Approximately $7-8 \mathrm{~mm}$ of the tips of each seminal vesicle is also harvested, as a separate noncancerous germline DNA specimen. Following the tissue harvest, the two prostate halves are resutured together using chromic suture or sealed together with special glue and then placed in $10 \%$ neutral buffered formalin for 1-3 days for fixation prior to sectioning.

\section{Parameters of pathological stage and prognosis}

Final pathologic stage, determined by examination of the RP specimen, predicts for likelihood of recurrence much more accurately than factors routinely available preoperatively-clinical stage, preoperative serum PSA, and biopsy Gleason score. In the Baylor RP series, prognostic factors that were independent predictors of prognosis by multivariate analysis included the level of the invasion of the cancer with respect to the prostatic capsule, and the status of the seminal vesicles, lymph nodes and surgical margins, as well as the Gleason score in the $\mathrm{RP}$ specimen.

\section{pT2a-b (Confined to the Prostate)}

A cancer completely confined to the prostate is defined as pT2a-b. The significance of the separate categories of pT2a (unilateral) and pT2b (bilateral) confined cancer is questionable because the majority of prostate cancers are multifocal. After RP, patients with pT2a-b cancer have an excellent prognosis with $>90 \%$ of patients PSA recurrence free at 5 years. At Baylor, we have subdivided patients with organ-confined PCa as level 0 (confined 


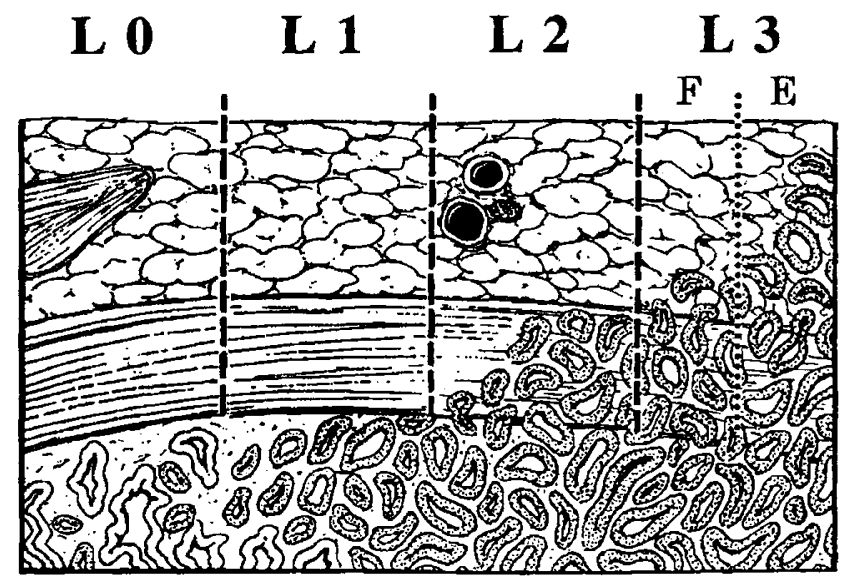

Figure 2 Levels of prostatic capsular invasion. Level 0 is tumor confined to the prostatic stroma, within the confines of normal glandular elements. Level 1 is tumor confined to the prostatic stroma, but beyond the boundary of normal glandular elements Level 2 is tumor confined to the prostate but within a layer more fibrous than muscular (capsule). Extraprostatic extension is designated as L3 and signifies extension of tumor into the periprostatic soft tissue, which may be classified as focal (L3F) or established (L3E). The sharpness of the boundaries between prostatic stroma, capsule, and adipose tissue is exaggerated for clarity.

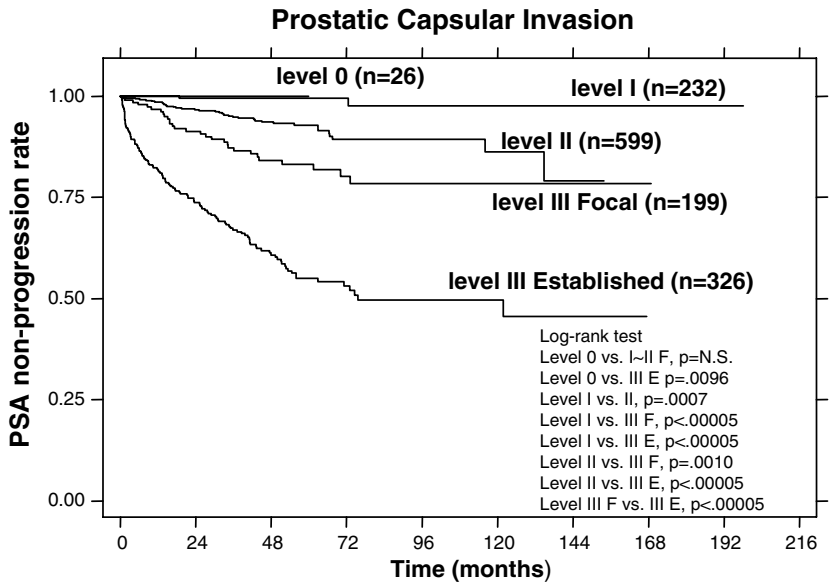

Figure 3 Kaplan-Meir disease-free probability plot by levels of capsular invasion.

to the prostatic stroma within the confines of the extent of normal prostatic acini), level 1 (confined to prostatic stroma but outside the extent of normal prostatic acini), and level 2 (into but not completely through the prostatic capsule, or at the boundary of the prostate at the apex or anteriorly where there is no capsule (Figure 2). ${ }^{15}$ Previously, we reported that patients with cancer confined to the prostate but with level 2 invasion of the prostatic capsule had a worse prognosis than patients with level 0-1 capsular invasion. None of 138 patients without capsular invasion had SVI or + LN suffered recurrence; all remained free of progression, even though some had large volume (up to $6.26 \mathrm{~cm}^{3}$ ) or poorly differentiated (Gleason sum up to 8) cancers. Invasion into the capsule $(n=271)$ was occasionally associated with SVI (6\%) or + LN (3\%) and a significantly lower PFP of $87 \%$ at 5 years. We confirmed these results in our updated prostatectomy series (Figure 3).

\section{pT3a (Extraprostatic Extension)}

A cancer extends through the prostatic capsule into the periprostatic adipose tissue is defined as pT3a tumor. These categories are further subdivided into 'focal' (L3F, only a few glands outside the prostate) and 'established' (L3E, anything more than focal) extraprostatic extension (EPE) (Figure 2). At Baylor, we use a more strict definition for L3F as not more than one high power field outside the prostate on more than two separate sections. Epstein has stated that our definition is too restrictive, in that a number of our patients would be considered as focal EPE according to their criteria, but are categorized as established EPE by us. ${ }^{16}$ In support of his opinion, in our series, the prognosis of patients with L3F is more similar to L2 patients than to L3E patients (Figure 3). Spread of tumor into the periprostatic fat is associated with a progressively increased risk of SVI and + LN. In a multivariate analysis of the Baylor series, the level of PCI was an independent prognostic factor $(P<0.001)$ and there was a strong association between the level of prostate capsular invasion (PCI) and the total tumor volume, Gleason grade, SVI, + LN and rate of biochemical recurrence after radical prostatectomy. Prostate cancer does not appear to metastasize in the absence of invasion into the capsule (or to the boundary of the prostate) regardless of the volume or grade of the intraprostatic tumor. Stratification of patients according to the level PCI may provide valuable prognostic information, although the reproducibility of this classification has not been studied. However, at Baylor we routinely report the level of PCI for each cancer focus.

\section{pT3b (Seminal Vesicle Invasion)}

The definition of SVI is that a cancer extends into the muscular layer of the seminal vesicle. Prostate cancer that involves the seminal vesicles has a poor prognosis generally. Patients with SVI not only have an increased incidence of nodal metastases, but the prognosis is worse for patients with SVI, even in the absence of $+\mathrm{LN}$ (Figure 4). We previously reported the mechanisms of SVI (Figure 5). ${ }^{17}$ Type I is direct spread along the ejaculatory duct complex into the seminal vesicles. Tumor migration does not occur in the lumen of the ejaculatory ducts; rather it invades along the tissue plane around these structures. Type II is spread across the base of the prostate, either directly across the tissue plane between the base of the prostate and the intraprostatic portion of the seminal vesicle (Type IIa) or through the fat between the base of prostate and the seminal vesicles (Type IIb). The 
Pathological Stage

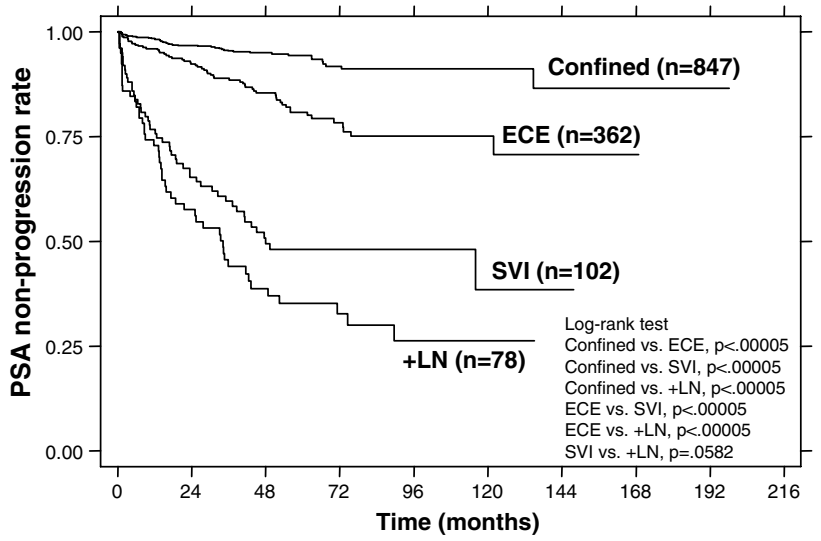

Figure 4 Kaplan-Meir disease-free probability plot by pathologic stage.

\section{Patterns of SVI}

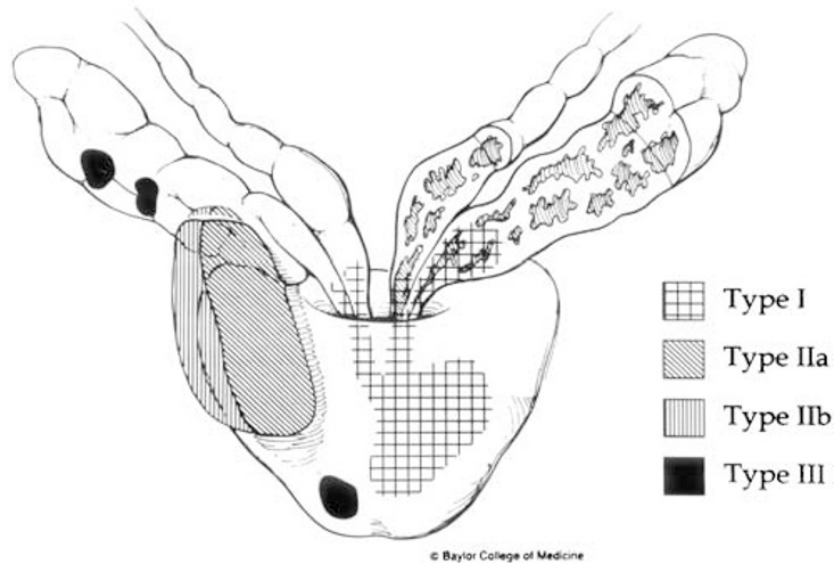

Figure 5 Three patterns of seminal vesicle involvement (SVI) and peri-SVI (see text).

presence of isolated foci of cancer in the seminal vesicles without direct continuity with the primary tumor characterizes Type III involvement, the least common mechanism of involvement. Although we originally reported a better progression-free survival for patients with type III SVI, in our recent analysis, controlling for other pathological parameters, this was no longer of independent prognostic significance. However, when we analyzed patients with SVI but without established EPE into the periprostatic fat, we found that these patients had an excellent prognosis, the same as otherwise similar patients without SVI. ${ }^{18}$ Many of these patients had only minimal involvement of the seminal vesicles, or involved only a portion of the seminal vesicles that is at least partially intraprostatic. The recent series from Hopkins has confirmed the better prognosis of these two subsets of patients. ${ }^{19}$ As a result of these findings, they do not classify patients with involvement of the intraprostatic portion of the seminal vesicles as positive for SVI. We would disagree with this approach, because once the ejaculatory duct divides into a paired, separate duct system, by definition, the medial duct is the vas deferens and the lateral duct is the seminal vesicle. However, based upon these recent studies, we now add a note to the report to indicate that such patients with intraprostatic SVI have a much more favorable prognosis than the usual patient with SVI.

\section{pT4 (Bladder Neck Invasion)}

The invasion of PCa into the bladder neck (BN) has been defined as T4, according to the UICC/AJCC staging manual. ${ }^{20}$ However, this clinical staging system was based upon the urologist finding gross invasion of the $\mathrm{BN}$ or external sphincter, which would elevate the patient to clinical stage IV disease. The significance of only microscopic involvement of these structures is controversial, as to whether it should be considered just pT3a or pT4. Of note, the College of American Pathologists practice protocol on prostate specifically states that only microscopic involvement of the BN should not be considered as pT4. ${ }^{21}$ In support of this view, a recent combined study of patients at Memorial Sloan Kettering Cancer Center and Baylor College of Medicine has clearly indicated that the microscopic BN invasion was not an independent predictor of PSA progression after RP, and the prognosis of patients with $\mathrm{BN}$ invasion was more dependent on other pathological features such as EPE, SVI, $+\mathrm{LN}$, and + SM. Moreover, the prognosis of patients with microscopic bladder neck invasion was more similar to pT3 PCa than to pT4 PCa; therefore, we do not recommend that such patients be considered pT4. The urologist should carefully consider the clinical findings when the pathologic stage is reported as pT4.

\section{Surgical margin status}

Positive surgical margins $(+\mathrm{SM})$ are generally considered to indicate that the cancer has not been completely excised and is now considered to be one of the most important prognostic parameters. In RP specimens, the presence of tumor cells at the inked margin of resection is considered to represent $+\mathrm{SM}$. Mazzucchelli et $a l^{22}$ have classified $+\mathrm{SM}$ as focal, extensive or equivocal. ${ }^{22}$ They define a focal positive margin as involving only one gland in only one of the step sections, with anything greater considered extensive. A validated and reproducible system to quantify a + SM would be useful, in that this is probably the most common reason for the urologist to call the pathologist after receipt of the pathology report.

The site of the + SM is frequently at the same site as the area of EPE; however, a + SM may result from capsular incision into an otherwise confined focus of PCa. A + SM without EPE at the site of the + SM is not infrequently seen, having been reported in 
from 9 to $62 \%$ of cases of $+\mathrm{SM}$ in the literature (Table 3). Some have preferred to designate a + SM in the absence of EPE anywhere in the gland as pT2X, because extraprostatic tumor at the site of the + SM cannot be excluded. However, we have preferred to classify such patients as pT2, but noting the presence of a $+\mathrm{SM}$.

Leibovich et $a l^{23}$ at the Mayo Clinic studied 76 patients with a single focus of + SM without EPE and who also had an adjuvant radiation therapy within 3 months after the operation. The PSA nonprogression rate at 5 years for these patients was $88 \%$, compared to $59 \%$ of the patients matched by age, the site of + SM, ploidy status, PSA, and Gleason score who were not given adjuvant radiation therapy. Therefore, they concluded that an adjuvant radiation therapy may be beneficial for such patients with a single + SM without EPE. In our series, however, PSA nonprogression rate for 57 patients with + SM without EPE was $89 \%$, so that we consider that only a single $+\mathrm{SM}$ without EPE may not indicate the necessity of an immediate adjuvant radiation therapy. A prospective randomized clinical trial is needed to answer this important question.

Table 3 Incidence of positive surgical margins without extraprostatic extension

\begin{tabular}{|c|c|c|}
\hline & $\begin{array}{l}\text { No. of patients with } \\
+S M\end{array}$ & $\begin{array}{c}\% \text { of }+S M \text { without } \\
E P E\end{array}$ \\
\hline Stamey et $a l^{26}$ & 83 & 62 \\
\hline Ackerman et al & 51 & 34 \\
\hline Conrud et al & 28 & 45 \\
\hline Watson et al & 99 & 9 \\
\hline Ohori et $a l^{17}$ & 78 & 29 \\
\hline Baylor & 140 & 32 \\
\hline MSKKCC & 29 & 48 \\
\hline Total & 509 & 35 \\
\hline
\end{tabular}

In a recent series of 1389 patients treated with radical prostatectomy, + SM had an impact on PSA nonprogression rate over the spectrum of pathologic stages, including pT2 (confined) cancer (Figure 6). PSA nonprogression rate at 5 years for patients with EPE (pT3a) with positive + SM was $50 \%$, compared to $80 \%$ of patients with EPE and $-\mathrm{SM}(P<0.0005)$. The location of the site of + SM is shown in Table 4 . Nearly $50 \%$ of + SM cases occurring in patients with PCa confined to the prostate had +SM recognized in the apical section. Patients with $+\mathrm{SM}$ at the base of the prostate had a worse prognosis compared to an anterior + SM. Interestingly, in Cox hazard regression analyses for the various models, $+\mathrm{SM}$ in the apical shave was the only significant predictor of PSA progression $(P=0.0021)$ when other established pathological features and serum PSA level were considered.

\section{Other prognostic factors Gleason grading system}

The most widely used and generally accepted grading scheme for prostatic adenocarcinoma was developed by Dr Donald Gleason, who was the referee pathologist for the Veterans Administration studies of PCa three to four decades ago. ${ }^{24}$ This system relies on architectural details of the individual cancer glands under low-to-medium magnification without specific regard to the cytologic features seen on high magnification. The architectural grade tends to parallel the cytologic grade. Because prostate cancer is usually heterogeneous, Gleason incorporated both a primary (most prevalent) and secondary (next most prevalent) grade in his system. He described five major patterns exist on a scale from 1 to 5 , well to poorly differentiated. Both the primary and secondary patterns are assigned a grade, and the primary grade is added to the secondary grade to obtain the

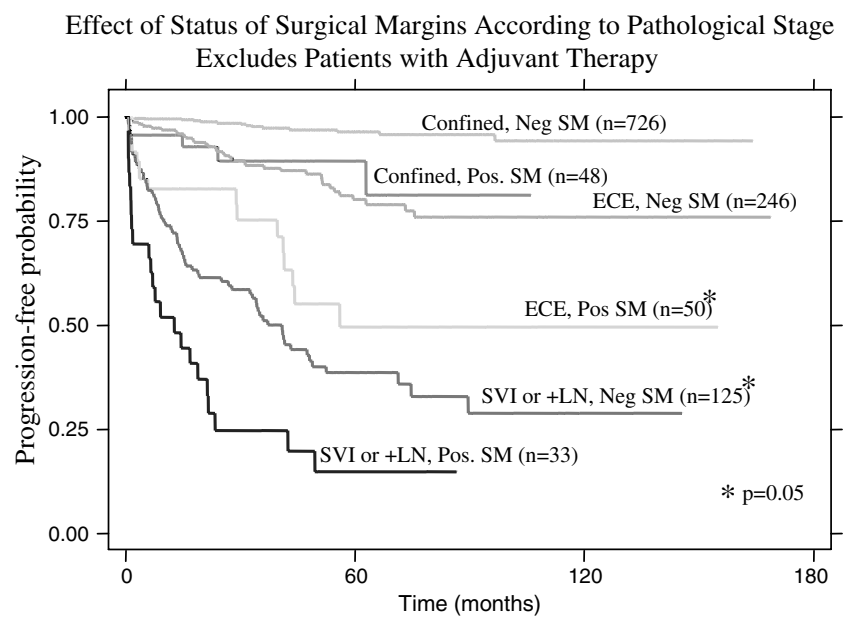

Figure 6 Kaplan-Meir disease-free probability across pathologic stages by surgical margin status, excluding patients who received adjuvant therapy prior to clinical or biochemical recurrence. 
Table 4 Location of positive surgical margins in RP spoecimens

$\%$ Location

\begin{tabular}{|c|c|c|c|c|c|c|c|c|}
\hline & \multirow[b]{2}{*}{ No. of $+S M$} & & & & & & & \\
\hline & & Apical & Anterior & Lateral & Posterior & Posterolateral & Bladder neck & Other \\
\hline Voges et al & 8 & 37 & 37 & - & - & - & 25 & - \\
\hline Rosen et al & 27 & 33 & 18 & 4 & 11 & 33 & - & - \\
\hline Epstein et al & 190 & 22 & - & - & 17 & 14 & 6 & - \\
\hline Stamey et al & 32 & 69 & - & - & - & 6 & - & 25 \\
\hline Van Poppel et al & 50 & 34 & - & - & - & 54 & - & 12 \\
\hline Watson et al & 90 & 38 & 11 & - & 26 & 17 & 9 & - \\
\hline Gomez et al & 22 & 46 & - & - & 14 & - & 14 & 27 \\
\hline \multicolumn{9}{|l|}{ Baylor (Maru et al) } \\
\hline With ECE & 84 & 25 & 31 & 4 & 14 & 17 & 9 & - \\
\hline Without ECE & 25 & 52 & 12 & 8 & 4 & 24 & - & - \\
\hline
\end{tabular}

Gleason sum, which could range from $2(1+1)$ to $10(5+5)$. One major criticism of the Gleason grading system is the limited recognition of the importance of the proportion of the tumor which is poorly differentiated (Gleason pattern 4 or 5). Nonetheless, reproducibility and reliability are satisfactory among those with training in genitourinary pathology. ${ }^{25}$ As the tumor becomes more poorly differentiated, the likelihood of disease recurrence increases. In contemporary series of patients who are selected for curative local therapy, few patients have well (Gleason sum 2-4) or poorly (Gleason 8-10) differentiated cancers. Most (85\%) are intermediate grade (Gleason 5-7), so that the broad scale of the Gleason spectrum provides limited discrimination in the typical patient.

It has been reported that \%Gleason grade $4 / 5$ cancer in prostatectomy specimens is the most significant predictor of PSA progression among a postoperative parameters. Recently, we studied 431 patients who had tumor maps with each Gleason grade and found that \%Gr $4 / 5$ (or Gr4/5 cancer volume) was an independent predictor of progression, as previously stated by the Stanford group, ${ }^{26}$ but it was not superior to the standard Gleason score in a multivariate analysis. Therefore, we concluded that it is not necessary to measure \%Gleason grade $4 / 5$ as standard practice. Importantly, however, patients with Gleason grade $4 / 5$ cancer between 1 and $5 \%$ had a worse prognosis than patients with no Gr4/5, but would not have been discriminated in the standard Gleason grade, because this requires that the secondary pattern be at least $5 \%$ of the volume of that tumor focus.

\section{Perineural invasion in RP specimens}

Perineural invasion (PNI) by PCa is seen in RP specimens in $75-84 \%$ of cases. In a series of $\mathrm{RP}$ reported 30 years ago, Byar and Mostofi $^{27}$ stated that PI, although helpful in making a diagnosis of prostate cancer in difficult cases, was not important prognostically. ${ }^{27}$ Although no data were presented to support this latter statement, it remained a dictum in pathology until relatively recently. Villers et $a l^{28}$ investigated PNI that was localized selectively to the area where nerves penetrate the prostate capsule, suggesting that PNI may facilitate extraprostatic spread. Bastacky et $a l^{29}$ revived interest in the prognostic significance of PNI in their study of needle biopsy specimens. They identified PI in $20 \%$ of cases and this finding was associated with a sensitivity of $27 \%$ and a specificity of $96 \%$ for predicting EPE in the prostatectomy specimen. The positive predictive value was $93 \%$ and the negative predictive value was $37 \%$. A multivariate analysis was not done. More recently, Egan and Bostwick ${ }^{30}$ analyzed the needle biopsy specimens of 349 men who underwent RP and found PI in $38 \%$ of their needle biopsy accessions. This more recent cohort also included many clinical T1c patients, in contrast to those studied by Bastacky which were all clinical T2 patients. Although PI was a prognostic factor in univariate analysis in the Mayo study, it was not so in a multivariate analysis. Only serum PSA, proportion of the biopsy involved by cancer, and Gleason score were independent predictive factors. Bonin et $a{ }^{31}{ }^{31}$ in a study of 484 patients with clinically localized PCa treated with three-dimensional conformal radiotherapy and followed for a median of 28 months, found that the presence of perineural invasion on the needle biopsy was associated with a significantly higher incidence of disease progression.

Ravery et $a l^{32}$ and Van den Ouden et al reported that PNI in RP specimens predicts progression as demonstrated with univariate analysis. However, neither of these studies showed significance by multivariate analysis. Because Gleason grade, pathologic stage, and tumor volume are well-established prognostic factors, many pathologists find little reason to note the presence of PNI in the RP specimen. Additionally, the assessment of PNI has not been accepted by the Cancer Committee of the College of American Pathologists. However, in our review of a consecutive series of RP specimens, we observed that increasing diameter PI space invasion was related to an increased likelihood of biochemical failure after RP. For purposes of this study, the 


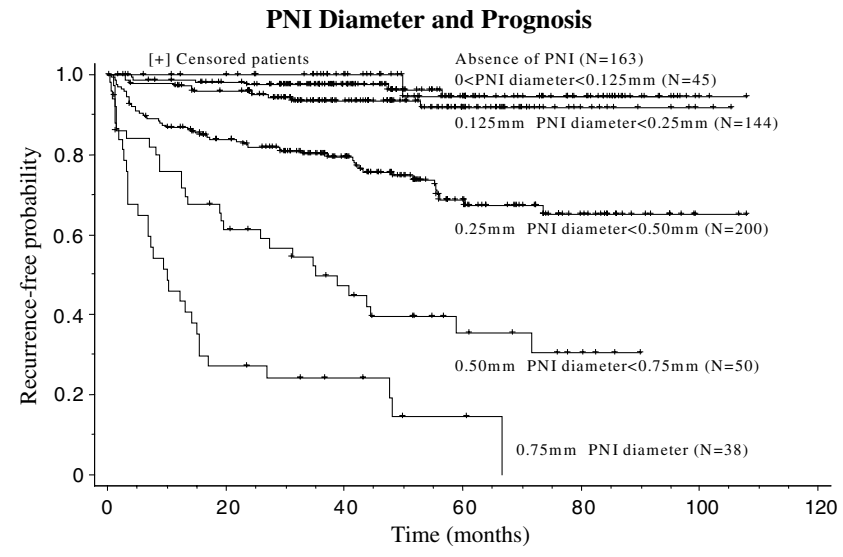

Figure 7 Kaplan-Meir disease-free probability across pathologic stages by greatest single diameter of Perineural space invasion in the RP specimen.

diameter of the greatest PNI focus in the prostatectomy specimen (either within or outside the prostate) was measured in a line perpendicular to the long access of the Schwann cell nuclei of the affected nerve. The prognostic significance of these findings was significant in univariate and multivariate analysis (Figure 7). In our study, we also demonstrated that large volume PNI was an important predictor of tumor progression, even in patients with cancer confined to the prostate (Figure 7). ${ }^{33}$ However, in a similar type of analysis of 414 patients with systematic biopsy, PNI in the biopsy specimen was not a significant predictor of PSA progression in a multivariate analysis. This may be explained by sampling error, because only $24 \%$ of PNI in RP specimens was sampled by the systematic biopsy. However, PNI in biopsy specimens was a significant predictor of the presence and laterality of ECE in RP specimens.

\section{Zonal distribution of cancer in RP specimens}

We studied retrospectively the clinical and pathological features of 1148 consecutive patients with prostate cancer treated with RP and followed with for a mean of 42 months. We categorized the patients with a large peripheral zone (PZ) cancer $\left(\geq 0.5 \mathrm{~cm}^{3}\right)$ with or without a small $\left(<0.5 \mathrm{~cm}^{3}\right)$ transition zone (TZ) cancer as 'predominantly PZ' cancer, and a large TZ cancer with or without a small PZ cancer as 'predominantly TZ', a large cancer in both PZ and TZ as 'Both' cancer. A small cancer in both PZ and TZ was classified as a 'Small' cancer. A PZ and TZ cancer focus was identified in 96 and $53 \%$ of the patients, respectively. If $\mathrm{PZ}$ cancer was present, $51 \%$ of the patients also had TZ cancer and if TZ cancer present, $94 \%$ of the patients also had PZ cancer. The median of the total $(\mathrm{PZ}+\mathrm{TZ})$ cancer volume was $1.62 \mathrm{~cm}^{3}(0-25.1), 1.07 \mathrm{~cm}^{3}(0-25.1)$ and $0.015 \mathrm{~cm}^{3}$
(0-18.1), respectively. With our definition, $59 \%$ of the patients had predominantly PZ cancer, $11 \%$ had predominantly TZ cancer, $10 \%$ had both and $20 \%$ had a small cancer. The frequency of predominantly PZ cancer decreased significantly over time $(70 \%$ before 1994 to $50 \%$ after 1997) and predominantly TZ cancer and small cancer significantly increased over the time. Only $37 \%$ of the TZ cancers had poorly differentiated elements (Gleason score $\geq 7$ ) and $36 \%$ extended outside of the prostate, compared to 64 and $51 \%$, respectively, for PZ cancer $(P<0.0001$ for both). The median of total tumor volume of predominantly PZ, predominantly TZ and both was $1.83,2.45$, and $3.58 \mathrm{~cm}^{3}$, respectively. Only five (4\%) patients of 129 with predominantly TZ cancer progressed within 5 years, compared to $20 \%$ with predominantly $\mathrm{PZ}$ cancer $(P<0.001)$ and $21 \%$ with both $\mathrm{PZ}$ and TZ cancer $(P<0.001)$ (Figure 9). With a Cox hazard regression model, the zonal distribution (small, TZ, both and PZ) was an independent predictor of PSA progression (HR $0.35,95 \%$ CI $0.14-0.86$ ), $P=0.023$ ) as well as other established prognostic factors. We concluded that the prognosis of a patient with prostate cancer is significantly more dependent upon the features of cancer in the PZ than in the TZ. Assessment of zonal distribution of prostate cancer is an important feature to gauge prognosis.

\section{Tumor volume}

Tumor volume can be measured most accurately with computerized planimetric methods, although a far simpler 'grid' method has been described. ${ }^{34}$ Of note, tumor volume in RP specimens has significantly decreased over the time. In recent years, the median of tumor volume was small, $<1 \mathrm{~cm}^{3}$. Although total tumor volume is one of the most important predictors of prognosis and is correlated with other pathologic features, in our series and the Hopkins series it was not an independent predictor of PSA progression when controlling for the other features of pathologic stage. Our results are different from the Stanford series, however, in that series the authors analyzed tumor volume of the most significant cancer focus, rather than the total tumor volume. At any rate, it does not appear necessary to measure tumor volume in routine clinical practice. However, tumor volume is still an important factor to predict for prior to biopsy, at least concerning 'insignificant' cancer, since tumor volume is one of the criteria in this definition.

\section{Intraductal cancer}

High-grade prostatic intraepithelial neoplasia (HPIN) is the only widely accepted precursor lesion for prostate cancer. However, the spread of established cancer within prostatic ducts may be indistinguishable morphologically from HPIN. By 
convention, all cytologically malignant cellular proliferations within prostatic ducts have been lumped into a PIN category, although there have been recent attempts by McNeal and others to develop reproducible criteria to separate high-grade HPIN from the spread of established prostate cancer within prostatic ducts-intraductal carcinoma (IDCa). Using McNeal's criteria for IDCa, we studied whole-mount sections from 252 patients with pT3No cancer for the presence of IDCa and correlated the presence or absence of IDCa with Gleason score, total tumor volume, surgical margin status, seminal vesicle involvement, and disease progression. ${ }^{35}$ Patients with IDCa had higher Gleason score and total tumor volume and were more likely to show seminal vesicle involvement and disease progression than those patients without IDCa. In addition, IDCa was of independent prognostic significance.

Rubin et $a \mathrm{I}^{36}$ also studied the $114 \mathrm{RP}$ to assess the prognostic significance of high-grade cribriform prostatic intraepithelial neoplasia (HGCP) and cribriform carcinoma (CC). They defined the patients into three categories: (1) pure acinar carcinoma: infiltrating acinar carcinoma without evidence of cribriform neoplasia; (2) CC: acinar carcinoma with CC; and (3) HGCP: acinar carcinoma with HGCP. HGCP was defined as the presence of neoplastic cells spanning the entire lumen in a cribriform configuration in which a basal cell layer could be shown by immunohistochemistry. Similar areas in which no basal cell layer could be seen were diagnosed as CC. The incidence of cribriform neoplasia was $38 \%$ (43 of 114). The incidence of HGCP and CC was 13\% (15 of 114) and 25\% (28 of 114), respectively. PSA nonprogression rate for patients with HGCP was $61 \%$ compared to $15 \%$ and $13 \%$ of patients with CC and pure acinar cancer, respectively $(P=0.0001)$. Both HGCP and CC are closely associated with several poor prognostic indicators, including advanced pathologic tumor stage, a high GS and serum PSA. Multivariate analysis showed HGCP as an independent prognostic indicator. The close association between high tumor volume and HGCP supports the theory that the development of HGCP is a late event in tumor progression, more compatible with the intraductal spread of tumor than dysplasia.

With the study of allelic instability, Dawkins et $a l^{37}$ reported that IDCa is not a simple extension of dysplasia, nor does it represent invasion of Gleason pattern 3 cancers into the ductal/acinar system. IDCa and Gleason pattern 4 cancer represent late but possibly separate events in prostate cancer evolution.

\section{Factors that determine prognosis and influence adjuvant therapy-preoperative and postoperative nomogram: (Figures 8 and 9)}

The presence of EPE itself does not necessarily mean an adverse prognosis since the patients with EPE alone without SVI, + LN, and + SM have a relatively high nonprogression rate. Also, patients with a poorly differentiated PCa have a better prognosis if the cancer is confined to the prostate. Although models exist that place patients into discrete groups at various risks for disease recurrence after surgery for $\mathrm{PCa}$, no single marker can distinguish those cancers which will recur vs those that will not recur in all patients. With the recent development of nomograms, which

\section{Preoperative Nomogram for Prostate Cancer Recurrence}

Points
PSA

Figure 8 Preoperative nomogram to predict recurrence. 
Postoperative Nomogram for Prostate Cancer Recurrence



Figure 9 Postoperative nomogram to predict recurrence.

are the result of a mathematical prediction model, it is possible to predict with reasonable accuracy the likely outcome of the patient based on multiple factors, utilizing parameters which are available preoperatively (preoperative nomogram) or postoperatively (postoperative nomogram). ${ }^{38,39}$ Most of the parameters in the last category are derived from information supplied by the pathologist. Because clinical stage and biopsy Gleason grade only approximate pathologic stage and Gleason grade in the prostatectomy specimen, prediction of prognosis should be more accurate when postoperative information is added to preoperative variables. By Cox proportional hazards regression analysis, Kattan et $a l^{38}$ modeled the clinical and pathologic data and disease follow-up for 996 men with clinical stage T1a-T3c NXM0 prostate cancer who were treated with RP by a single surgeon at Baylor College of Medicine. Prognostic variables included pretreatment serum PSA level, specimen Gleason score, presence and/or extent of prostatic capsular invasion, and the status of surgical margins, seminal vesicles, and lymph nodes. Validation was performed on this set of men and a separate sample of 322 men from five other surgeons' practices from that institution. The predictions from the nomogram appear to be accurate and discriminating, with a validation sample area under the receiver operating characteristic curve (ie, a comparison of the predicted probability with the actual outcome) of 0.89. As other prognostic factors are added to these nomograms in the future, the predictive accuracy of these models should increase.

\section{References}

1 Young HH. The early diagnosis and radical cure of carcinoma of the prostate: being of study of 40 cases and presentation a radical operation which was carried out in 4 cases. Bull John's Hopkins Hospital 1905; 16:315-321.

2 Walsh PC, Donker PJ. Impotence following radical prostatectomy: insight into etiology and prevention. J Urol 1982;128:492-497.

3 Hall GS, Kramer CE, Epstein JI. Evaluation of radical prostatectomy specimens. A comparative analysis of sampling methods. Am J Surg Pathol 1992;16: 315-324.

4 Schmid HP, McNeal JE. An abbreviated standard procedure for accurate tumor volume estimation in prostate cancer. Am J Surg Pathol 1992;16:184-191.

5 Grossfeld GD, Chang JJ, Broering JM, et al. Does the completeness of prostate sampling predict outcome for patients undergoing radical prostatectomy?: data from the CAPSURE database. Urology 2000;56:430-435.

6 Hollenbeck BK, Bassily N, Wei JT, et al. Whole mounted radical prostatectomy specimens do not increase detection of adverse pathological features. J Urol 2000;164:1583-1586.

7 Hollenbeck BK, Rubin MA. Prostate sampling to predict radical prostatectomy outcome. Urology 2001; 57:394-395.

8 True L. Surgical pathology examination of the prostate gland. Practice survey by American Society of Clinical Pathologists. 1994;102:572-579.

9 Mazzucchelli R, Montironi R, Preziozo D, et al. Surgical pathology examination of radical prostatectomy specimens. Updated protocol based on the Italian TAP study. Anticancer Res 2001;21:3599-3607.

10 Hollenbeck BK, Bassily N, Wei JT, et al. Whole mounted radical prostatectomy specimens do not 
increase detection of adverse pathological features. J Urol 2000;164:1583-1586.

11 Sehdev AE, Pan CC, Epstein JI. Comparative analysis of sampling methods for grossing radical prostatectomy specimens performed for nonpalpable (stage T1c) prostatic adenocarcinoma. Hum Pathol 2001;32:494-499.

12 Rosen MA, Goldstone L, Lapin S, et al. Frequency and location of extracapsular extension and positive surgical margins in radical prostatectomy specimens. J Urol 1992;148(Part 1):331-337.

13 Aihara M, Wheeler TM, Ohori M, et al. Heterogeneity of prostate cancer in radical prostatectomy specimens. Urology 1994;43:60-66, discussion 66-67.

14 Wheeler T, Lebovitz RM. Fresh tissue harvest for research from prostatectomy specimens. Prostate 1994;25:274-279.

15 Wheeler TM, Dillioglugil O, Kattan MW, et al. Clinical and pathological significance of the level and extent of capsular invasion in clinical stage T1-2 prostate cancer. Hum Pathol 1998;29:856-862.

16 Epstein JI. Pathologic assessment of the surgical specimen. Urol Clin North Am 2001;28:567-594.

17 Ohori M, Scardino PT, Lapin SL, et al. The mechanisms and prognostic significance of seminal vesicle involvement by prostate cancer. Am J Surg Pathol 1993;17:1252-1261.

18 Soh S, Arakawa A, Suyama K, et al. The prognosis of patients with seminal vesicle involvement (SVI) depends upon the level of extraprostatic extension (EPE). Abstract \#1141. J Urol 1998;159:296A Am J Clin Pathol 102: 572-579.

19 Epstein JI, Partin AW, Potter SR, et al. Adenocarcinoma of the prostate invading the seminal vesicle: prognostic stratification based on pathologic parameters. Urology 2000;56:283-288.

20 Fleming ID, Cooper JS, Hensen DE, et al. AJCC Cancer Staging Manual. 5th edn. Philadelphia: LippincottRaven Publishers, 1997.

21 Srigley JR, Amin MB, Bostwick DG, et al. Updated protocol for the examination of specimens from patients with carcinomas of the prostate gland: a basis for checklists. Cancer Committee. Arch Pathol Lab Med 2000;124:1034-1039.

22 Mazzucchelli R, Montironi R, Preziozo D, et al. Surgical pathology examination of radical prostatectomy specimens. Updated protocol based on the Italian TAP study. Anticancer Res 2001;21:3599-3607.

23 Leibovich BC, Engen DE, Patterson DE, et al. Benefit of adjuvant radiation therapy for localized prostate cancer with a positive surgical margin. J Urol 2000;163:1178-1182.

24 Gleason DF. Classification of prostatic carcinomas. Cancer Chemother Rep 1966;50:125-128.

25 Allsbrook W, Mangold K, Johnson M, et al. Interobserver reproducibility of Gleason grading of prostatic carcinoma: urologic pathologists. Hum Pathol 2001;32:74-80.
26 Stamey TA, McNeal JE, Yemoto CM, et al. Biological determinants of cancer progression in men with prostate cancer. JAMA 1999;281:1395-1400.

27 Byar DP, Mostofi FK. Carcinoma of the prostate: prognostic evaluation of certain pathologic features in 208 radical prostatectomies. Examined by the stepsection technique. Cancer 1972;30:5-13.

28 Villers A, McNeal JE, Redwine EA, et al. The role of perineural space invasion in the local spread of prostatic adenocarcinoma. J Urol 1989;142:763-768.

29 Bastacky SI, Walsh PC, Esptein JI. Relationship between perineural tumor invasion on needle biopsy and radical prostatectomy capsular penetration in clinical stage B adenocarcinoma of the prostate. Am J Surg Pathol 1993;17:336-341.

30 Egan AJ, Bostwick DG. Prediction of extraprostatic extension of prostate cancer based on needle biopsy findings: perineural invasion lacks significance on multivariate analysis. Am J Surg Pathol 1997;21: 1496-1500.

31 Bonin SR, Hanlon AL, Lee WR, et al. Evidence of increased failure in the treatment of prostate carcinoma patients who have perineural invasion treated with three-dimensional conformal radiation therapy. Cancer 1997;79:75-80.

32 Ravery V, Boccon-Gibod LA, Meudemans A, et al. Predictive value of pathological features for progression after radical prostatectomy. Eur Urol 1994;26: 197-201.

33 Maru N, Ohori M, Kattan MW, et al. Prognostic significance of the diameter of perineural invasion in radical prostatectomy specimens. Hum Pathol 2001;32:828-833.

34 Humphrey PA, Vollner RT. Percentage carcinoma as a measure of prostatic tumor size in radical prostatectomy tissues. Mod Pathol 1997;10:326-333.

35 Wilcox G, Soh S, Chakraborty S, et al. Patterns of highgrade prostatic intraepithelial neoplasia associated with clinically aggressive prostate cancer. Hum Pathol 1998;29:1119-1123.

36 Rubin MA, Dunn R, Kambham N, et al. Should a Gleason score be assigned to a minute focus of carcinoma on prostate biopsy? Am J Surg Pathol 2000;24:1634-1640.

37 Dawkins HJ, Sellner LN, Turbett GR, et al. Distinction between intraductal carcinoma of the prostate (IDC-P), high-grade dysplasia (PIN), and invasive prostatic adenocarcinoma, using molecular markers of cancer progression. Prostate 2000;44:265-270.

38 Kattan M, Eastham J, Stapleton A, et al. A preoperative nomogram for disease recurrence following radical prostatectomy for prostate cancer. J Natl Cancer Inst 1998;90:766-771.

39 Kattan MW, Wheeler TM, Scardino PT. Postoperative nomogram for disease recurrence after radical prostatectomy for prostate cancer. J Clin Oncol 1999;17: 1499-1507. 